\title{
Mechanism of ice nucleation in liquid water on alkali feldspars $\uparrow$
}

\author{
Alice Keinert, ${ }^{a}$ Kathrin Deck, + $^{a}$ Tilia Gaedeke, ${ }^{a}$ Thomas Leisner (D) ab \\ and Alexei A. Kiselev (iD *a
}

Received 5th December 2021, Accepted 11th January 2022

DOI: $10.1039 / \mathrm{d} 1 \mathrm{fd} 00115 \mathrm{a}$

Crystallization of supercooled liquid water in most natural environments starts with heterogeneous nucleation of ice induced by a nucleation site. Mineral surfaces, which form the majority of aqueous interfaces in Earth's ecosystem, possess a plethora of surface morphological and chemical features that can serve as ice nucleation sites. The nature of surface sites responsible for ice nucleation from supersaturated water vapor have been recently identified for alkali feldspar, a family of rock-building minerals constituting $60 \%$ of the Earth's crust. It was demonstrated that ice preferentially forms upon the patches of crystalline surface with (100) orientation, exposed in the surface defects such as cracks, pores, and pits arising due to chemically induced stress and further enhanced by hydrothermal alterations of natural feldspars. However, whether the same sites were responsible for nucleation from liquid water, remained to be shown. Here, we investigate the mechanism of heterogeneous ice nucleation in a layer of aqueous sucrose solution on top of thin sections of feldspar prepared along the (010) crystalline plane. We observe a preferential orientation of ice crystals defined by an epitaxial relationship between feldspar and ice, with ice crystals growing on the crystalline surfaces of feldspar with (100) orientation. We thus conclude that the ice nucleating sites active in deposition freezing mode are also active in the immersion freezing regime. This conclusion is further supported by the enhancement of ice nucleation active site density observed for the thin sections of feldspar prepared sub-parallel to the (100) plane as compared to sections prepared along (010) and (001) crystallographic orientations.

\section{Introduction}

Although water in crystalline form constitutes less than $2 \%$ of the total water reservoir on Earth, almost $70 \%$ of the world's fresh water exists in the form of

\footnotetext{
${ }^{a}$ Karlsruhe Institute of Technology, Institute of Meteorology and Climate Research, Karlsruhe, Germany. E-mail: alexei.kiselev@kit.edu

${ }^{b}$ Institut für Umweltphysik, Universität Heidelberg, Heidelberg, Germany

$\dagger$ Electronic supplementary information (ESI) available. See DOI: 10.1039/d1fd00115a

$\$$ Now at TU Delft, Faculty of Aerospace Engineering, Department of control and operations, Delft, The Netherlands.
} 
snow and ice. ${ }^{1}$ Crystallization of supercooled water is one of the main mechanisms responsible for precipitation formation in atmospheric clouds, and as such is important for the Earth's water cycle and for the cloud-feedback as part of the global radiation budget. ${ }^{2-4}$ Thus, the importance of water-ice transformation for the Earth's ecosystem cannot be overestimated.

In the atmosphere, cloud droplets can be supercooled to a temperature as low as approximately $-38{ }^{\circ} \mathrm{C}$ before spontaneous nucleation of an ice embryo leads to the crystallization of water. At temperatures below $-25{ }^{\circ} \mathrm{C}$ formation of ice may happen directly from supersaturated water vapor via deposition on the surface of aerosol particles. This latter mechanism was shown to be efficient if mineral dust particles are present in the air, acting as ice nucleating particles (INP).

Even though the composition of mineral dust is highly heterogeneous, ${ }^{5}$ not all minerals are equally efficient INPs. It was recently demonstrated that certain types of alkali feldspar are capable of triggering ice nucleation at temperatures just below the melting point, ${ }^{6-8}$ with the mineralogy of feldspar apparently being a decisive factor for its ice nucleating efficacy. The highly ice nucleation (IN) active types of alkali feldspars feature micron-scale lamellar intergrowth of more K-rich and more Na-rich alkali feldspar, known as perthitic intergrowth. It was suggested that ice nucleates preferentially at the sites associated with microstructural defects (the so-called nanochannels and pull-aparts) forming due to the tensile stress near the intergrowth boundaries. ${ }^{9}$

In our previous study of ice crystals nucleation and growth on natural perthitic alkali feldspars, the preferential epitaxial nucleation of ice by the alignment of the ice (1010) prism plane with (100) faces of the feldspar was identified as the mechanism associated with high IN activity of alkali feldspar in deposition freezing mode. ${ }^{10}$ Similar results have been reported in a later experimental study conducted with different feldspar samples under similar conditions, ${ }^{\mathbf{1 1}}$ but could not be reproduced by molecular dynamics (MD) simulation performed using the (100) surfaces of an idealized K-feldspar crystal. ${ }^{12}$ To explain how the noncleavable crystalline (100) face becomes accessible for ice nucleation, we hypothesized that small patches of (100) become exposed in the cracks and steps that are characteristic for feldspar cleavage surfaces (001) and (010). This hypothesis has received experimental support in the more recent study demonstrating enhancement of the IN activity of K-rich feldspar caused by chemicallyinduced fracturing. ${ }^{13}$ Preferential nucleation of ice in surface pits and cracks has been reported repeatedly for immersion ${ }^{\mathbf{1 4 , 1 5}}$ and deposition freezing, ${ }^{\mathbf{1 0 , 1 1}}$ however, no direct evidence that ice nucleation is associated with (100) surfaces in both freezing mechanisms has been provided so far.

Recent studies of deposition freezing suggest that the presence of liquid water either adsorbed on the mineral surface ${ }^{16}$ or condensing in pores and cavities via capillary condensation is required for initial ice nucleation, ${ }^{17,18}$ which would imply that the same IN active sites should be responsible for freezing under both mechanisms. And yet, the direct evidence that the patches of (100) are responsible for ice nucleation in liquid water were missing. ${ }^{19}$ In this communication we report the results of ice nucleation experiments in concentrated sucrose solution in contact with feldspar samples, prepared as thin sections parallel to the three main crystallographic orientations (001), (010) and (100). We observe a preferential orientation of ice crystals nucleating heterogeneously on feldspar allowing us to deduce the nature of the IN active sites. We address the question, if the frequency 
of appearance of the (100) patches is associated with the feldspar microtexture, by comparing the ice nucleating efficacy of two distinctly different feldspars: a perthite microcline feldspar with rich microtexture and an almost pure K-rich adularia orthoclase feldspar, devoid of any visible microtexture.

\section{Materials and methods}

\section{Sample preparation}

Specimens of two natural feldspars have been used in this study. The first one is a natural perthitic alkali feldspar from Pakistan (samples prepared from this specimen are labelled FS06). Visually, the centimetre-sized specimen with partially developed facets is pale beige and turbid (see ESI Fig. S1A $\dagger$ ). The X-ray powder diffraction analysis (XRD, Panalytical, Cu K-alpha 1\&2) yielded a phase content of $41 \%$ orthoclase, $39 \%$ microcline, and $20 \%$ albite (see Table ST1 $\dagger$ for details). FS06 has a complex microperthitic structure overlain by polysynthetic twinning according to the pericline law $^{20}$ and porosity, very likely related to hydrothermal or deuteric alteration ${ }^{21}$ (see Fig. S2 $\dagger$ ). The fully coherent exsolution lamellae are characteristic for slowly cooled microcline feldspar ${ }^{21}$ and are oriented so that the lattice misfit between the more K-rich and more Na-rich regions is minimized. This condition defines the so-called Murchison plane, ${ }^{22,23}$ which has non-rational Miller indices between $(\overline{6} 01)$ and $(\overline{8} 01)$. We use this fact for identification of crystallographic orientations in the samples prepared from the FS06 specimen.

The second specimen (labelled FS07) is a K-rich sanidine feldspar (adularia) from Austria. The FS07 contains over 90\% pure orthoclase phase (see ESI Table $\mathrm{ST} 1 \dagger)$ and is almost transparent. Apart from few macroscopic cracks, FS07 does not exhibit any microtexture at any scale, neither appearing in polarization microscope nor in the scanning electron microscope imaging.

For this study, both specimens were cut along the main crystallographic plains (001), (010) and (100) and the thin sections parallel to these planes were prepared following standard petrographic sample preparation techniques. The crystallographic orientations of the thin sections parallel to the cleavage planes (010) and (001) were later confirmed by backscattered electron diffraction (Hikari Super BSED system, AMETEK EDAX) and analysed using TSL OIM 8 software. The orientation of the (100) surface was deduced from the known geometric relationship to the cleavage planes. The deviation between the actual crystallographic plane and the orientation of the thin section was estimated at below $2^{\circ}$ for the cleavage planes but could be as large as $\pm 10^{\circ}$ for the thin sections prepared along (100).

For comparison, the fragments of both specimens were ground in a ceramic mortar and sieved through a $20 \mu \mathrm{m}$ sieve. The powders of FS06 and FS07 were then used for preparation of aqueous suspensions with various concentrations of solid material for the droplet freezing assay (DROFA) experiment.

\section{Sucrose solution experiment}

Nucleation and growth of ice in supercooled water is too fast to be observed with the usual means and requires high-speed video-recording techniques to capture the initial stage of ice formation. ${ }^{14}$ However, the growth rate of ice crystals can be 
significantly slowed down in a concentrated solution where water diffusivity is significantly reduced. In 1957, Bigg $^{24}$ introduced a method of counting airborne ice crystals by allowing them to settle onto the surface of a supercooled sugar solution. We use this approach to investigate the kinetics of crystal nucleation and growth on the surface of thin sections of feldspar.

In this study, a small droplet (approximately $1 \mu \mathrm{L}$ volume) of $60 \mathrm{wt} \%$ (weight $\%$ ) aqueous solution of sucrose (Merck) was placed on the surface of a sample thin section and covered with a microscope cover slide to avoid evaporation (Fig. S3†). The sample was positioned on the Peltier stage mounted on the rotation table of the polarization microscope (Leica DM4P), and the whole microscope was enclosed into an air-tight flexible glove box filled with water-free synthetic air to avoid water condensation and frost formation on the cold surfaces. The Peltier stage was slowly cooled down to $-30{ }^{\circ} \mathrm{C}$ or until several ice crystals became visible in the field of view of the $5 \times$ objective piece. The temperature was then manually adjusted to the apparent melting point of ice in the sucrose solution (between $-13{ }^{\circ} \mathrm{C}$ and $-15{ }^{\circ} \mathrm{C}$ ) to slow down or completely stop the growth of ice crystals. In several cases the temperature was slightly raised to achieve partial melting of ice crystals allowing for observation of the dendrite geometry and identification of crystallographic axes of individual ice crystals. We refer to such crystals as being "optically thinned".

\section{DROFA measurements}

Thin sections. The ice nucleating efficacy of the thin sections and powder suspensions of feldspar samples was measured in the DROFA setup as described in our earlier work., ${ }^{8,13}$ Briefly, a $10 \times 10 \mathrm{~mm}$ thin section was placed into a temperature-controlled cold stage setup consisting of a Linkam MDBCS-196 motorized cold stage, a piezo-driven drop-on-demand dispenser (GeSIM, model A010-006 SPIP) and a video camera with a wide-field objective allowing for detection of individual freezing events with $0.125 \mathrm{~s}$ time resolution. In the experiments described in this work, 50 to 150 droplets of pure water each with a volume of approximately $1.4 \mathrm{~nL}$ were deposited onto the polished surface of a thin section in a square pattern with $400 \mu \mathrm{m}$ centre-to-centre separation between the droplets. During the freezing experiments the cold stage was cooled with a rate of $3 \mathrm{~K} \mathrm{~min}^{-1}$. Time and temperature of freezing of every individual droplet were recorded automatically and stored in the form of the temperature dependent fraction of frozen droplets (freezing spectra) for further analysis.

Suspension droplets. Approximately 70 droplets with a volume of $21.6 \mathrm{~nL}$ containing $0.01 \mathrm{wt} \%, 0.1 \mathrm{wt} \%$, or $1 \mathrm{wt} \%$ suspensions of feldspar particles were deposited in a $9 \times 9$ array with a PipeJet Nano dispenser (BioFluidix $\mathrm{GmbH}$ ) on a clean $10 \times 10 \mathrm{~mm}$ silicon wafer (Ted Pella, Inc.). Freezing of the suspension droplets was measured using the DROFA technique as described above. The specific surface area (SSA) of the feldspar powders was determined using an Autosorb iQ model 7 gas sorption system (Quantachrome Instruments, a brand of Anton Paar QuantaTec Inc.). Using argon as a sorbent gas at the boiling point of 87 $\mathrm{K}$ and applying Brunauer-Emmett-Teller (BET) theory, ${ }^{25}$ the SSA of $(2.5 \pm 0.2) \mathrm{m}^{2}$ $\mathrm{g}^{-1}$ was found for the FS06 and $(1.8 \pm 0.2) \mathrm{m}^{2} \mathrm{~g}^{-1}$ for the FS07 powder samples.

The heterogeneous freezing efficacy of a substrate was expressed in terms of Ice Nucleation Active Site (INAS) surface density, ${ }^{26} n_{\mathrm{s}}(T)$, as a function of 
temperature $T$ using the fraction of frozen droplets $f_{\text {ice }}(T)$ and the total area of ice nucleating particles contained in a single droplet $S_{\mathrm{d}}$. In the DROFA experiments with suspension droplets the $S_{\mathrm{d}}$ is calculated from the SSA of the powder and the weight concentration of feldspar material in a suspension droplet. For droplets deposited on a thin section, $S_{\mathrm{d}}$ is the area of contact between the pure water droplet and the substrate. In this way, the IN efficacy of the powder samples and thin sections can be compared in a quantitative way.

ESEM IN experiments. An environmental scanning electron microscope (ESEM, FEI Quattro FEG, Thermo Fisher Scientific) was used to investigate the surface morphology of feldspar samples and to conduct a limited series of deposition ice nucleation experiments following the method described in our previous work. ${ }^{10}$ Using the modified cooling specimen stage of the microscope, the feldspar sample was cooled down to $-28{ }^{\circ} \mathrm{C}$ with the rate of $-1 \mathrm{~K} \mathrm{~min}^{-1}$ to achieve ice nucleation and growth. The overall pressure in the ESEM specimen chamber was kept at $450 \mathrm{~Pa}$ (humidified $\mathrm{N}_{2}$ gas) with the partial water pressure set to about $50 \mathrm{~Pa}$, which is just above the saturation pressure with respect to ice but below the water saturation pressure at this temperature. Sample cooling was terminated once ice crystals were visually detected on the surface, thus allowing them to develop well-defined pristine crystal habits.

\section{Results and discussion}

\section{Ice crystals growing in sucrose solution}

Thin section FS06. The main result of this study is that the ice crystals, heterogeneously nucleating in aqueous sucrose solution in contact with the (010) thin section of FS06 feldspar, would grow in a preferential orientation defined by the epitaxial relationship between the crystal structures of feldspar and ice (Fig. 1). The apparent habit of the ice crystals (rectangular envelope filled with branching dendrites) requires some explanation which is given below.

At very small supercooling (below $1 \mathrm{~K}$ ), ice crystals in aqueous solutions grow preferentially along the basal plane, forming first platelets and then flat dendritic crystals as growth proceeds. ${ }^{27}$ At moderate supercooling the dendrites become inclined to the basal plane, with the inclination angle being a function of supercooling temperature and solution concentration. ${ }^{28,29}$ At a supercooling of $5 \mathrm{~K}$ below the melting point of ice, the primary dendrites split into two symmetric segments, forming three-dimensional hollow hexagonal pyramids joined at their apices. ${ }^{29-31}$ The secondary dendrites would then branch off at angles defined by the crystal structure of ice and the splitting angle. Similar shapes have been reported by Pruppacher, ${ }^{27}$ Macklin and Ryan, ${ }^{31}$ and Knight and DeVries, ${ }^{29}$ where a thorough discussion of the observed growth behaviour can also be found.

The sucrose solution in our experiments forms a thin layer (about $0.2 \mathrm{~mm}$ ) on the surface of feldspar, and thus the growth of ice crystals is constrained in the direction perpendicular to the sample surface. This constraint is not only due to the confinement, but also because the solution is cooled through the substrate, and thus the growth of ice away from the surface is less favourable. In our experiments we observe ice crystals assuming two stable orientations: with the basal plane either parallel or perpendicular to the plane of confinement. If the ice crystals grow with their basal plane parallel to the substrate, their habit exhibits clearly hexagonal symmetry, although the dendrite branches become curved in 


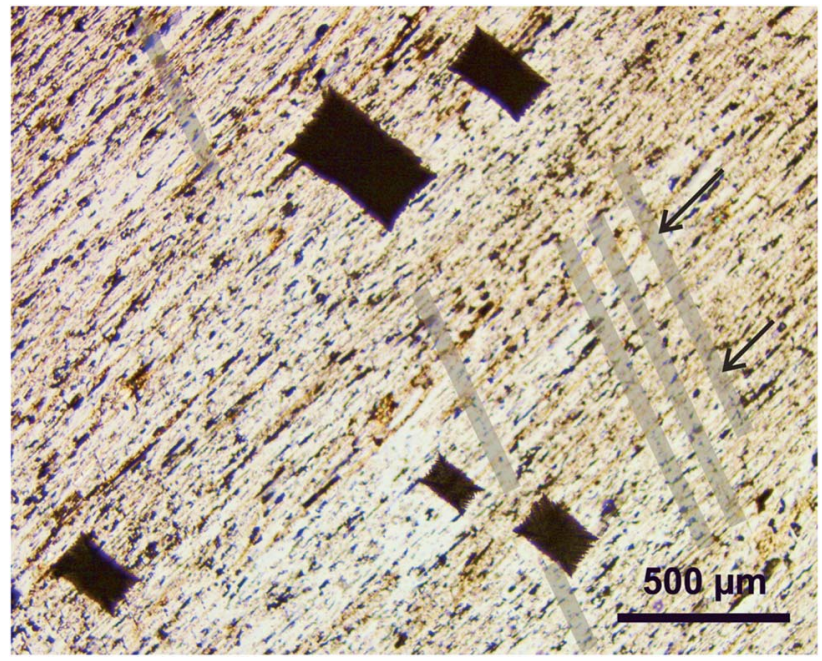

Fig. 1 Ice crystals growing in sucrose solution on top of FS06 thin section along the (010) crystallographic plane of feldspar. The grey bands are manually overlaid on top of the (001) cleavage cracks to guide the eye. The rectangular shape of the crystals is explained in the text. Note the identical orientation of the ice crystals.

response to the asymmetrical temperature gradients in the liquid,,$^{32}$ as shown by the ESI Fig. S7. $\dagger$ In the latter case, the major axis of ice ( $C$-axis) lies in the plane of the substrate, but the full 3D pyramid cannot develop in the lateral plane because the growth of a basal plane is constrained in the direction perpendicular to the sample surface. As a result, the ice crystals have the habit of inverse double pyramid cut along the prismatic plane. Fig. 2 shows a structure of the ice crystal

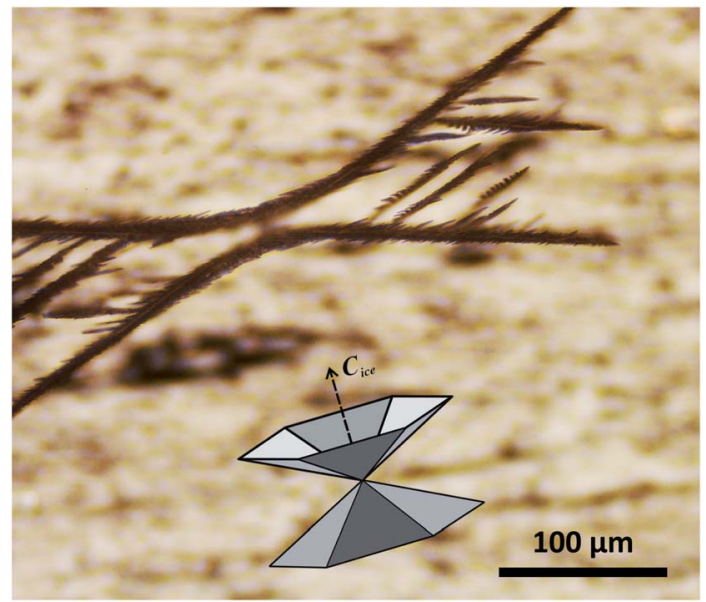

Fig. 2 3D-dendrite structure of an ice crystal in equilibrium with sucrose solution at $-14.2^{\circ} \mathrm{C}$. The overlay shows the structure of the inverse double pyramid of ice redrawn after Knight and DeVries, ${ }^{29}$ with the orientation of the $C$-axis of the hexagonal ice crystal indicated by the arrow. 
stabilized shortly after nucleation by adjusting the temperature to the melting point of ice in the sucrose solution at $-14.2^{\circ} \mathrm{C}$. The idealized structure of the inverse double pyramid is overlaid on the image to illustrate the spatial arrangement of the crystallographic axes of the ice. Interestingly, Holden and coauthors $^{\mathbf{1 4 , 3 3}}$ recently published similar images of rectangular ice crystal shapes appearing in pure water in contact with a thin section of microcline feldspar polished along the (010) cleavage plane.

In order to establish the geometrical relationship between the orientation of ice and crystalline structure of feldspar, we notice two morphological features visible on the surface of the thin section: (a) the cleavage cracks and (b) the exsolution lamellae. Feldspar has two perfect cleavage planes along (010) and (001) with approximately $90^{\circ}$ angle between them,,$^{34}$ and in a bulk crystal of natural feldspar cleavage cracks would appear along these planes if mechanical stress (as a result of thermal expansion or chemical alteration) is applied. On a thin section with orientation (010) as the one shown in Fig. 1, cracks would be aligned with the orthogonal cleavage plane (001). To aid the eye, the cleavage cracks are overlaid with transparent bands in Fig. 1. ESI Fig. S4A $\dagger$ gives a clearer view of a series of cracks on the same substrate.

Orientation of cleavage cracks together with the orientation of the thin section (010) allow for unambiguous identification of the crystallographic orientation of the FS06 feldspar specimen (010). A projection of the unit cell of feldspar linked to the orientation of cleavage cracks is shown in Fig. 3.

Comparing the orientation of ice with the orientation of the feldspar unit cell in the (010) thin section (Fig. 3), it becomes obvious that the primary prismatic plane $(10 \overline{1} 0)$ of ice crystals must be aligned with the (100) plane of feldspar in such a way that the directions of the main crystallographic axes of both crystals coincide (see also ESI Fig. S4B†). This is only possible if ice grows epitaxially on

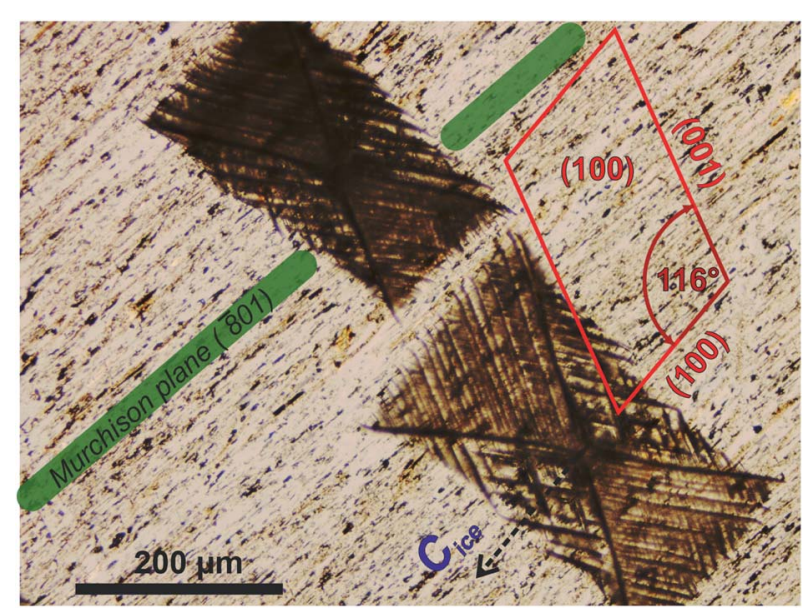

Fig. 3 Oriented ice crystals growing in sucrose solution in contact with FS06 thin section with orientation of (010). Projection of feldspar unit cell identified from the orientation of cleavage cracks and exsolution lamellae is shown in red. Orientation of Murchison plane (601) is highlighted in green, while the direction of the main crystallographic axis of the ice is indicated by the broken arrow line. 
the (100) surfaces of feldspar, implying that these surfaces must be the nucleation sites of ice. This is in agreement with our previous finding ${ }^{10}$ that ice crystals growing from water vapor would be nucleating on the patches of (100) plane exposed in cracks and steps present on the surface of natural perthitic feldspars. Such surface defects are present in abundance on the surface of the (010) thin section, and are associated with the boundaries between exsolution lamellae, as illustrated by the SEM image of the FS06 (010) sample shown in Fig. S5a. $\dagger$ In contrast, the surface of FS07 samples is almost devoid of any surface topography, as illustrated by ESI Fig. S5b. $\dagger$

The exsolution lamellae are stretched along the Murchison plane having the Miller indices between ( $\overline{8} 01)$ and $(\overline{6} 01)$ and is thus sub-parallel to (100) with the angle between $8^{\circ}$ and $11^{\circ}$. The Murchison plane is described by non-rational Miller indices and does not, in general, coincide with any of the primary atomic planes in feldspar. ${ }^{22,23}$ In practice that means that the orientation of exsolution lamellae can be anywhere between planes with Miller indices of ( $\overline{6} 01)$ and $(\overline{801})$. We investigate the relationship between the ice crystal orientation (which we assume is aligned with the (100) plane of feldspar) and the orientation of exsolution lamellae. Fig. 4 shows the frequency distribution of the angles. From the 87 crystals observed in all IN experiments with the sucrose solution in contact with FS06 thin section (010), approximately 60 were in the range between $5^{\circ}$ and $11^{\circ}$ with the maximum at $8.1^{\circ}$. For the microcline feldspar with the lattice parameters defined in the ESI Table ST1, $\uparrow$ this value is exactly equal to the angle between the planes with Miller indices ( 801$)$ and (100) (which is equal to $8.13^{\circ}$ ), thus nicely confirming our hypothesis that ice crystals are epitaxially nucleated and grown on the sites with (100) orientation. The variability of $\pm 10^{\circ}$ (Fig. 3) comes mostly from the irregular form of exsolution lamellae, which complicates the identification of the Murchison plane.

Interestingly, we could not observe the oriented growth of ice in sucrose solution in contact with other thin sections prepared from the same specimen. Ice crystals growing in all possible orientations were observed on the thin sections with orientations parallel to the (001) and sub-parallel to the (100). However, in most cases ice was growing with its basal face parallel to the substrate. One

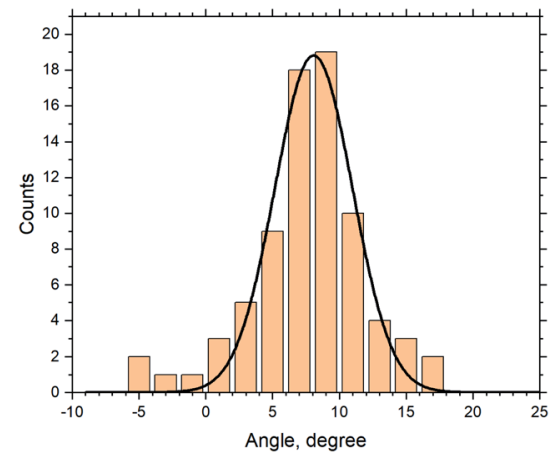

Fig. 4 Distribution of angles between the main crystallographic axis of ice and the preferential orientation of exsolution lamellae within the (010) plane of FS06. The distribution is fitted with a Gauss curve (the black line) with maximum at $8.1^{\circ}$. 
possible reason for such behaviour could be that the only sample where ice crystals would have their $c$-axis exactly parallel to the surface is the thin section parallel to the (010). In all other cases, the ice crystals would have to grow with their basal plane tilted with respect to the sample surface. Such geometry, which is easily achievable for deposition growth of ice (as demonstrated in ESI Fig. S6†), could be unfavourable for ice growth in a confined liquid layer. The same argument applies to the thin section of FS06 which was prepared sub-parallel to (100): since the alignment is not perfect, the patches with (100) orientation would be inclined with respect to the surface resulting in an inclination between the basal face of ice and the sucrose solution layer.

Furthermore, no orientational growth of ice could be observed on any of the thin sections of the adularia feldspar FS07. We explain this observation by the lack of morphological features where the patches of (100) could have been exposed. This is consistent with our previous observation of absence of orientational growth of ice on the sanidine feldspar in the deposition mode. ${ }^{\mathbf{1 0}}$

Deposition IN experiments. This study was focused on immersion freezing and therefore only a limited series of experiments with the FS06 sample has been performed. These experiments, conducted in the ESEM in an atmosphere of humidified nitrogen gas, have revealed preferential orientation of ice crystals growing upon the cleaved (001) surface of the FS06 specimen (see ESI Fig. S6†). As in our earlier work, ${ }^{10}$ pristine ice crystals with identical orientation were nucleating at a temperature of $-28{ }^{\circ} \mathrm{C}$ at the vapor pressure below saturation with respect to water, which is traditionally described as the deposition freezing regime. The tilt angle and the orientation of ice crystals with respect to the exsolution lamellae and the cleavage cracks suggest that the nucleation of ice crystals occurred epitaxially on the patches with (100) orientation. We thus conclude that the ice nucleating sites active in deposition and in the immersion freezing modes have the same crystallographic nature. In both freezing mechanisms, patches of feldspar surface with (100) orientation must be responsible for ice nucleation.

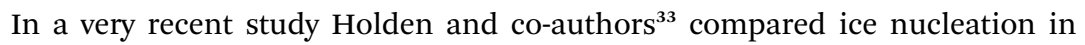
immersion and deposition mode by using high-speed video imaging. They observed oriented growth of ice crystals on a polished section of feldspar from water vapor and explained it by an epitaxial relationship between the crystalline structure of feldspar and ice. As the orientation of ice crystals was apparently in agreement with that observed in immersion freezing experiments, a common mechanism of ice nucleation in both modes has been suggested. However, no correlation between the sites of initial ice nucleation in the two freezing regimes could be established.

Droplet freezing assay measurement results. The DROFA measurements have been performed with the thin sections polished along the two cleavage planes of feldspar specimens (001) and (010), and a thin section that was prepared along the (100) plane according to geometry considerations. The results of these measurements are shown in Fig. 5 and 6, together with the results for the aqueous suspensions of powder samples in three different weight concentrations. For comparison, the parameterization of the INAS density of a reference feldspar ${ }^{6}$ is shown as a straight dashed line.

The first glance at the freezing behaviour of both specimens reveals that droplets in contact with FS06 freeze at $10 \mathrm{~K}$ higher temperature than for the FS07, 


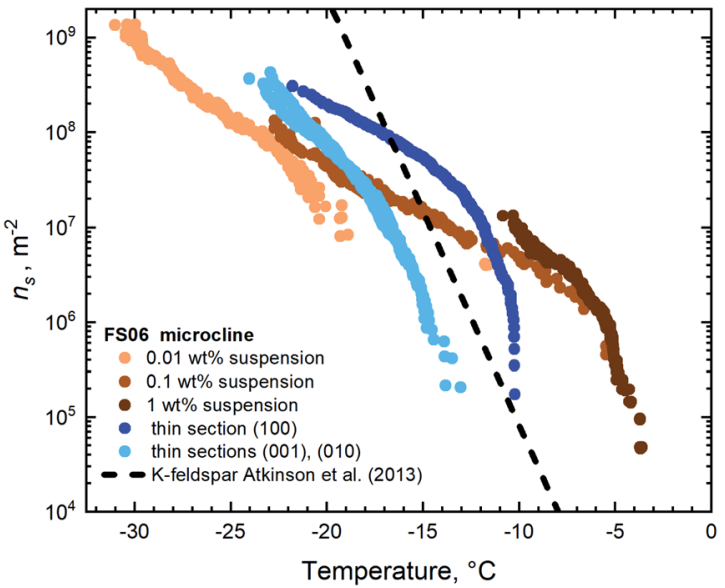

Fig. 5 IN active sites surface densities on the thin sections and for the suspensions of FS06 feldspar.

which we do not find surprising given the well-established empirical relationship between the feldspar microtexture and its IN efficacy. ${ }^{7-9,13}$ What is indeed noteworthy is that the droplets are freezing at almost $5 \mathrm{~K}$ higher temperature on the surfaces of a thin section polished along the (100) plane as compared to other orientations. This result is somewhat unexpected given the absence of oriented ice growth in sucrose solution in contact with the same surfaces. As the preparation of a thin section along a non-cleavable plane is connected with certain practical difficulties (starting with but not limited to the orientation uncertainty), we have not expected such a striking difference in IN efficacy of the thin sections with different crystallographic orientations. The plausible explanation of this result is that the patches of surface with (100) orientation should appear more frequently in the steps and pits present on the surface of this thin section. The

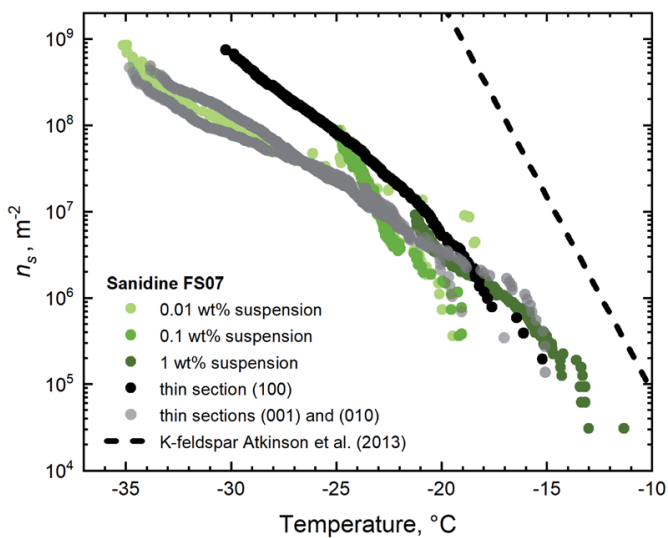

Fig. 6 IN active sites surface densities on the thin sections and for the suspensions of FS07 sanidine feldspar. 
absence of apparent epitaxial growth in the sucrose solution experiment is likely due to the mismatch between the preferential plane of ice crystal growth and the plane of confinement defined by the solution layer, as discussed above.

The thin section with (100) orientation shows the highest IN efficacy for both FS06 and FS07 specimens, although the relative enhancement in the case of FS07 is smaller and only pronounced below $-20^{\circ} \mathrm{C}$. Assuming that the mechanism responsible for the enhanced ice nucleation is the same (exposure of the surface patches with (100) orientation), the smaller variation of the $n_{\mathrm{s}}(T)$ is expected, given the relative homogeneity and reduced density of surface topography features on the polished surfaces of the FS07 samples as compared to FS06.

We also note a good general agreement between $n_{\mathrm{s}}(T)$ values obtained for pure water droplets in contact with the thin sections parallel to (001) and (010) and for aqueous suspensions. For FS06, however, the number of high-temperature active sites (which are active above freezing temperature of $-10{ }^{\circ} \mathrm{C}$ ) in the powder sample is significantly higher than on the thin section sample with highest IN efficacy (the one sub-parallel to (100)). We hypothesize that such hightemperature sites might have been created by grinding the bulk specimen, thus allowing access to more IN active sites per unit surface area. Similar conclusions have been reached by Holden and co-authors. ${ }^{14}$ Indirectly, this hypothesis goes along with our recent observation that chemically induced cracks in the initially texture-free sanidine feldspar (similar to FS07) indeed increase its IN efficacy. ${ }^{13}$ No such high-temperature IN active sites have been found in the samples prepared from the FS07 specimen.

Finally, the similarity of $n_{\mathrm{s}}(T)$ values for the thin sections with (001) and (010) orientation observed for both samples FS06 and FS07 should be noted. The values for both orientations are identical within the experimental uncertainties on the order of $100 \%$, typical for droplet freezing assay experiments. Within the suggested mechanistic explanation, this is not surprising for high-temperature IN efficacy of the FS06 specimen given the similar surface density of exsolution lamellae exposed on both the cleavage planes. This, however, cannot explain the identity of $n_{\mathrm{s}}(T)$ values observed for the whole temperature range from -35 to $-10{ }^{\circ} \mathrm{C}$ for both the FS06 and FS07 samples, as patches of crystalline plane parallel to (100) are rare in FS06 and absent in FS07. We thus have to assume that the reason for identical IN efficacy of the orthogonal cleavage planes lies in the similarity of lattice constants and surface energies of these planes. ${ }^{34}$ For FS07 samples, the close match between the $n_{\mathrm{s}}(T)$ values of cleavage planes and aqueous suspensions suggests that mechanical grinding of the crystalline specimen exposes predominantly surfaces coinciding with the cleavage planes, whereas in the ground FS06 sample crystalline surface parallel to the (100) dominates the freezing behaviour at higher temperature.

\section{Conclusions}

In summary, we have applied a well-established experimental technique initially devised 70 years ago for detection and counting of airborne ice-crystals to investigate the nature of ice nucleating active sites associated with the microcline feldspar. The technique relies on the drastically reduced growth rates of ice in the concentrated (60 wt\%) aqueous solution of sucrose, allowing for observation of crystal habits evolution in real time without the necessity to use high-speed 
recording methods. By observing the ice crystal growth in a thin layer of sucrose solution in contact with the (010) thin section of microperthitic feldspar, we were able to establish a relationship between the lattice structure of feldspar and the crystalline structure of ice. The ice crystals were preferentially oriented in a way that their main crystal axis is parallel to the main crystallographic axis of feldspar. This is only possible if ice crystals nucleate and grow epitaxially with their prismatic crystal planes $(10 \overline{1} 0)$ attached to the feldspar crystal surface with (100) orientation. Such surfaces must be exposed in the cavities, steps, and pits which are characteristic for the exsolved alkali feldspars. The evolution of a perthite microstructure in alkali feldspar, often enhanced by hydrothermal/deuteric alteration, fosters the exposure of (100) crystal surfaces and thus enhances the IN activity of exsolved alkali feldspars. This conclusion is strongly supported by the results of the droplet freezing assay study. Here, we have shown that the IN efficacy of the microperthitic microcline FS06 is significantly higher than that of the sanidine sample FS07, which is devoid of cracks, inclusions or perthitic structure. The IN efficacy of the thin section prepared sub-parallel to the (100) orientation was further enhanced in comparison to other orientations, supposedly due to the higher surface density of the crystal surfaces with the true (100) orientations.

Comparing the results of ice nucleation experiments in the aqueous sucrose solutions with the previous experiments conducted with feldspar samples in deposition freezing mode, we arrive at the conclusion that the nature of the most active ice nucleating sites in both processes must be the same. In both cases, the crystal surfaces with (100) orientation must be responsible for the onset of ice nucleation. Considering recent doubts of the validity of the deposition freezing concept, the identity of IN active sites in both types of experiments reported here supports the conclusion that the so-called deposition freezing always requires formation of liquid water in contact with active sites to initiate heterogeneous freezing. The fact that deposition freezing is observed below water saturation, should be explained in view of the recently suggested pore condensation and freezing (PCF) mechanism. The surface topography of the samples investigated in this study fully justify this conclusion.

\section{Author contributions}

AAK conceived the idea of the study and led the manuscript preparation with contributions from all authors. AK conducted the droplet freezing assay experiment, evaluated the results and wrote the manuscript. KD and TG performed sucrose solution experiments, analysed the results and prepared the graphics for the manuscript. TL contributed to the conception of the experiment and provided the general supervision. All authors contributed to the discussion of the results.

\section{Conflicts of interest}

The authors declare no conflicts of interest.

\section{Acknowledgements}

The authors acknowledge financial support by the Helmholtz Association under Atmosphere and Climate Programme (ATMO). The authors also greatly 
acknowledge XRD analysis of feldspar powder samples performed by Dr Jörg Göttlicher (Institute of Photonic Science KIT-IPS). AAK acknowledges Prof. Rainer Abart from the Vienna University for his valuable help in interpretation of crystallographic relationships in feldspars.

\section{References}

1 S. H. Schneider, T. L. Root and M. D. Mastrandrea, Encyclopedia of Climate and Weather, Oxford University Press, 2011.

2 S. Bony, R. Colman, V. M. Kattsov, R. P. Allan, C. S. Bretherton, J.-L. Dufresne, A. Hall, S. Hallegatte, M. M. Holland, W. Ingram, D. A. Randall, B. J. Soden, G. Tselioudis and M. J. Webb, J. Clim., 2006, 19, 3445-3482.

3 O. Boucher, D. Randall, P. Artaxo, C. Bretherton, G. Feingold, P. Forster, V.-M. Kerminen, Y. Kondo, H. Liao, U. Lohmann, P. Rasch, S. K. Satheesh, S. Sherwood, B. Stevens and X.-Y. Zhang, in Climate Change 2013: the Physical Science Basis. Contribution of Working Group I to the Fifth Assessment Report of the Intergovernmental Panel on Climate Change, ed. T. F. Stocker, D. Qin, G.-K. Plattner, M. Tignor, S. K. Allen, J. Boschung, A. Nauels, Y. Xia, V. Bex and P. M. Midgley, Cambridge University Press, Cambridge, 2014, ch. Clouds and Aerosols, pp. 571-658.

4 B. J. Murray, K. S. Carslaw and P. R. Field, Atmos. Chem. Phys., 2021, 21, 665679.

5 N. A. Marsden, R. Ullrich, O. Möhler, S. Eriksen Hammer, K. Kandler, Z. Cui, P. I. Williams, M. J. Flynn, D. Liu, J. D. Allan and H. Coe, Atmos. Chem. Phys., 2019, 19, 2259-2281.

6 J. D. Atkinson, B. J. Murray, M. T. Woodhouse, T. F. Whale, K. J. Baustian, K. S. Carslaw, S. Dobbie, D. O'Sullivan and T. L. Malkin, Nature, 2013, 498, 355-358.

7 A. D. Harrison, T. F. Whale, M. A. Carpenter, M. A. Holden, L. Neve, D. O'Sullivan, J. Vergara Temprado and B. J. Murray, Atmos. Chem. Phys., 2016, 16, 10927-10940.

8 A. Peckhaus, A. Kiselev, T. Hiron, M. Ebert and T. Leisner, Atmos. Chem. Phys., 2016, 16, 11477-11496.

9 T. F. Whale, M. A. Holden, A. N. Kulak, Y.-Y. Kim, F. C. Meldrum, H. K. Christenson and B. J. Murray, Phys. Chem. Chem. Phys., 2017, 19, 31186-31193.

10 A. Kiselev, F. Bachmann, P. Pedevilla, S. J. Cox, A. Michaelides, D. Gerthsen and T. Leisner, Science, 2017, 355, 367-371.

11 E. Pach and A. Verdaguer, J. Phys. Chem. C, 2019, 123, 20998-21004.

12 A. Soni and G. N. Patey, J. Chem. Phys., 2019, 150, 214501.

13 A. A. Kiselev, A. Keinert, T. Gaedeke, T. Leisner, C. Sutter, E. Petrishcheva and R. Abart, Atmos. Chem. Phys., 2021, 21, 11801-11814.

14 M. A. Holden, T. F. Whale, M. D. Tarn, D. O'Sullivan, R. D. Walshaw, B. J. Murray, F. C. Meldrum and H. K. Christenson, Sci. Adv., 2019, 5, eaav4316.

15 R. W. Friddle and K. Thürmer, Atmos. Meas. Tech., 2020, 13, 2209-2218.

16 A. Laaksonen and J. Malila, Atmos. Chem. Phys., 2016, 16, 135-143.

17 R. O. David, C. Marcolli, J. Fahrni, Y. Qiu, Y. A. Perez Sirkin, V. Molinero, F. Mahrt, D. Brühwiler, U. Lohmann and Z. A. Kanji, Proc. Natl. Acad. Sci. U. S. A., 2019, 116, 8184-8189. 
18 C. Marcolli, Atmos. Chem. Phys., 2020, 20, 3209-3230.

19 B. J. Murray, Science, 2017, 355, 346-347.

20 I. Parsons, P. Thompson, M. Lee and N. Cayzer, J. Sediment. Res., 2005, 75, 919940.

21 I. Parsons, Mineral. Mag., 2010, 74, 529-551.

22 J. D. Fitz Gerald, I. Parsons and N. Cayzer, Am. Mineral., 2006, 91, 772-783.

23 J. V. Smith and W. L. Brown, Feldspar Minerals, Springer, Berlin Heidelberg, 2nd edn, 1988.

24 E. K. Bigg, Tellus, 1957, 9, 394-400.

25 S. Brunauer, P. H. Emmett and E. Teller, J. Am. Chem. Soc., 1938, 60, 309-319.

26 G. Vali, Atmos. Chem. Phys., 2014, 14, 5271-5294.

27 H. R. Pruppacher, J. Glaciol., 1967, 6, 651-662.

28 C. Knight, Cryst. Growth Des., 2020, 20, 580-589.

29 C. A. Knight and A. L. DeVries, Phys. Chem. Chem. Phys., 2009, 11, 5749-5761.

30 W. C. Macklin and B. F. Ryan, J. Sediment. Res., 1965, 22, 452-459.

31 W. C. Macklin and B. F. Ryan, Philos. Mag., 1966, 14, 847-860.

32 C. A. Knight, J. Appl. Phys., 1962, 33, 1808-1815.

33 M. A. Holden, J. M. Campbell, F. C. Meldrum, B. J. Murray and H. K. Christenson, Proc. Natl. Acad. Sci. U. S. A., 2021, 118, e2022859118.

34 P. H. Ribbe, in Feldspars and Their Reactions, ed. I. Parsons, Springer Netherlands, Dordrecht, 1994, pp. 1-49. 